\title{
THINK SMART, THINK SOCIAL! THE ROAD MAP FROM SMARTER OBJECTS TO SOCIAL OBJECTS IN SOCIAL INTERNET OF THINGS - A SURVEY
}

\author{
A.Meena Kowshalya* \\ Government College of Technology, India \\ M.L.Valarmathi \\ ACCET, India
}

Internet of Things, the one paradigm many vision idea is ruling the world. By 2025 over trillions and trillions of objects will be connected to the internet. Social networking concepts are revolutions beyond IOT. One of the many visions of IOT is to make objects not only smarter but also socially conscious. A new paradigm named Social Internet of Things evolved which integrated two technologies namely Internet of Things and Social Networking. A SloT comprises of socially aware smart objects that can autonomously establish and enable collaboration with other smart objects that are friends. In this paper we study the role, characteristics of social objects and their relationships. Five kinds of relationships are identified. These relationship and characteristics helps in revealing the level of trust between objects. Experiments were conducted for 85 social objects in an office environment and the types of objects, their relationships, interest, activities etc were discovered.

Key words: Internet of Things, Smart objects, Social Internet of Things, Object relationships, Social awareness

\section{INTRODUCTION}

Internet of things is a novel paradigm that is based on pervasive presence of numerous things or objects. These things or objects include sensors, actuators, RFIDs, mobile phones etc., which collaborate among each other through addressing mechanisms with their friends and friend of friends to achieve a desired tasks. The future internet will embody millions of objects. IoT will provide the opportunities for users, manufacturers and service providers and make all real virtual. Bringing loT into real world can be possible through integration of several technologies. Few technologies include identification; sensing and communication technologies and SOA based architecture for middleware. IoT finds its application almost in all fields including transportation and logistics domain, healthcare domain, smart environment domain, personal and social domain. Much research in standardization activity, addressing and networking issues, security and privacy has made comfortable use of IoT services across the globe. The above mentioned aspects has diverted to a different thinking of loT ie., making loT sociable. One can think of a new vision of loT, making objects smarter and social.
Social Networking concepts can be used to provide social capabilities to objects in loT. This kind of thinking has led to a new paradigm known as Social Internet of Things (SloT) where objects are able to collaborate with each other autonomously via owners. These objects can crawl the billions of loT objects for discovering services. Social objects can also autonomously advertise themselves to the rest of the internet community. This new vision of loT called SloT is a 10 year old research area that has opened many research application areas. Primarily three kinds of objects can be identified.

i) Objects that have higher interoperability with external systems and collaborate with human social networks.

ii) Objects that interact with surrounding and exhibit partial pseudo social behavior with neighbors.

iii) Objects that are able to build their own social network and collaborate with other social objects.

This paper is organized as follows. Section 2 describes the key enabling technologies of loT, Section 3 introduces the concept of Social Internet of Things, and Section 4 presents the experimental setup followed by Conclusion. 


\section{IOT- ONE PARADIGM AND MANY VISIONS}

IoT had made anytime, anywhere, any how access of things. The facet includes many visions. These differences in the vision depend on interests of the stakeholders and their background. Today the numbers of objects connected to the internet are higher compared to the magnitude of the internet. We are in need of different technologies to interact with each other. The need for pervasive computing is more since computing and communication technologies will be embedded in our environments.

\section{Enabling technologies of IoT}

The key enabling technologies of loT described by:
i) Tagging Things
ii) Sensing Things
iii) Thinking things
iv)Shrinking Thinks

RFID and related technologies enable real time identification and tagging. Sensor technologies enable detection of environmental status and sensory information. Smart technologies build intelligence at the networks border. Nanotechnologies enable the networking of smaller and smaller things. The complete loT vision can be achieved only if objects are able to collaborate with each other in an open way and autonomously. Current implementations allow objects to collaborate only with small closed groups. Even small group collaborations need IPC gateways. Thus the number of embedded computing devices will increase in the environment leading to scalability problems. One solution to this issue is trusted social communities among objects. This can be achieved by Social Internet of Things. The social networking concept integrated along with loT has led to a new type of network which can be modeled with social behavior and social relationships between objects. Objects that are smarter and socially conscious build a SloT community [01].

\section{Smart objects}

Modern technologies have made smart objects available. [02] derives and analyzes the transformation of smart objects into socially conscious smart objects. Smart objects are considered as the building blocks of Internet of Things [03]and are classified according to their awareness, representation and interaction. Three main catego- ries were identified:

i) Activity aware smart objects: maintain logs of information about work activities of its own and others

ii) Policy aware smart objects: understand events and activities with respect to predefined policies.

iii) Process aware smart objects: understand inbuilt processes and provides context aware guidelines.

Recent smart objects also exhibit pseudo social behavior. The distinction between a "Thing" that is simply connected to an internet and a "Thing" that takes part in active role in the network has to be clearly defined. An acting object is an object that is able to translate the awareness of casual relationships into actions. An active object has the ability to stimulate action and participate in social web, having a self-confident role within the social web. [04 - 07] lists smart objects namely Smart-Its, Blog-jects, Embodied Micro blogging, spontaneous and their activities. Many unanswered problems exists in the loT arena. A few includes what really objects talk about, does these conversations are useful and do they promote developments for human society, should objects need a separate social network that of humans. The concept of social networks of IoT objects separated from that of a human but submissive to their need is supported by recent studies [08 - 11].

\section{SOCIAL INTERNET OF THINGS}

A novel paradigm of "social network of intelligence objects" based on the notion of social relationships among objects [12]. Objects establish social relationships with each other autonomously. These gave loT a structure that can be navigable, scalable and can perform efficient service discovery [13]. [14, 15] have studied SloT environment and has proved that the network is navigable and efficient service search can be performed. This enables to generate trust among objects to influence the level of interaction between Things that are friends. A social network allows people to increase popularity, find old friends, get filtered information and find new friends. Things in turn publish and find information and services, update services and get environmental characteristics. [16] has studied social virtual objects in the cloud.This is the right time to define kind of social relationship and 
behavior among objects. [18] proposed a community detection algorithm for the Social Internet of Things based on movement, preference and social similarity.

\section{Object relationships}

Five kinds of object relationships are identified [12]:

i) Parental object relationship

ii) Co location object relationship

iii) Co work object relationship

iv)Social object relationship

v) Owner object relationship

Parental object relationships are defined among similar objects build by the same manufacturer. Co location object relationship is determined whenever objects reside constantly at same place. Co work object relationship is defined as the relationship between objects when they come into contact at their owner's work place. Social object relationship is established when objects come into contact periodically or continuously for purely reasons related to relations among owners. Ownership object relationship is established when objects owned by the same user come into contact.

\section{Relation structures for social relations}

A widely accepted classification of social relations is proposed by [17] through his relation model theory. Four basic relational frames or structures are sources for generating social actions. These are derived from four elementary models of [17]. The relational frames are:

i) Community sharing

ii) Equality matching

iii) Authority ranking

iv)Market pricing
Communal sharing relationships can be associated with behaviors of objects which are not relevant individually but have a collective relevance. Communal sharing objects are associated to whole group. Equality matching relationship may represent all forms of information exchange between objects that operate as equals and that request and provide information amongst them in view of providing loT services to users while maintaining individuality. These objects associate to a service that it advertises. Authority ranking relationship is asymmetrical based on precedence, hierarchy, status, command and difference. They are established between objects of different kinds of complexity and hierarchal levels. The service advertised is associated to the whole group of objects or to the object of highest rank. Market pricing relationships are based on proportionality with interactions organized with reference to a common scale of ratio values. These can be associated with interactions that objects have whenever they find themselves having to work together in the view of achieving mutual benefit. Cooperation among smart objects iscrucial in many loT applications.

\section{EXPERIMENTAL RESULTS - CASE STUDY OF AN OFFICE ENVIRONMENT}

A total of 85 objects were used to study the social relationships and behavior of objects in an office. The office consisted of 50 PCs, 8 smart phones, 22 laptops and 5 tablets. All 50 PCs belong to the same manufacturer. Table 1 lists the type of devices that were available in the office. A total of 65 users were asked to make use of the objects. All objects were connected across social networking sites. The interests and activities of all objects were audited. Each device was initialized with social profiles of participants.

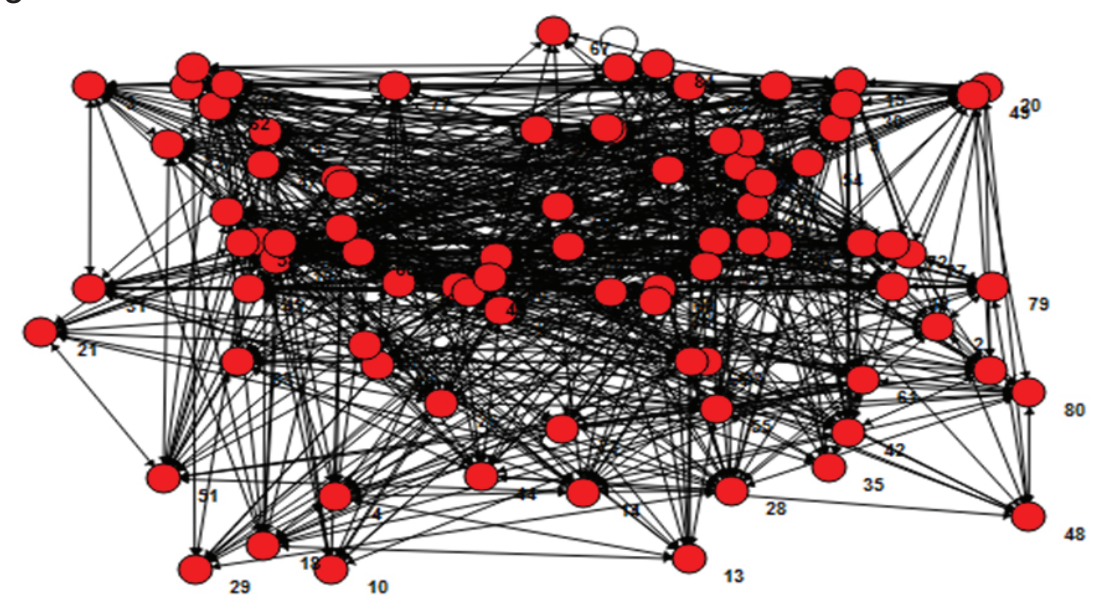

Figure 1: Layout of the Social graph 
Also the participants were asked to log on to Facebook in order to keep track of their friends and interests. The devices were kept powered on all the time. Figure 1 shows the social graph of the 85 users. The graph was generated by SocNetV1.9

Table 1: Total number of objects

\begin{tabular}{|c||c||c||c||}
\hline S.No & Objects/ Things & Manufacturer - number of devices & Total Objects \\
\hline \hline 1 & PCs & Dell - 50 & 50 \\
\hline \hline 2 & Smart phones & Samsung -5; Apple -3 & 8 \\
\hline \hline 3 & Laptops & Dell -15; Hp -7 & 22 \\
\hline \hline 4 & Tablets & Samsung - 5 & 5 \\
\hline
\end{tabular}

Each device performs a periodic Bluetooth device discovery every $100+/-10.11$ seconds for duration of $10.11 \mathrm{~s}$ to find out about nearby devices. The experimental hardware is an HTC s620 Windows Mobile Smartphone. HTC s620 has a $200 \mathrm{MHz}$ TI processor, 64MB of RAM, 128MB of $\mathrm{ROM}$ and a MicroSD slot. The radio interfaces include a quad-band GSM/EDGE cellular radio, Bluetooth $v 1.2$ and $802.11 \mathrm{~b} / \mathrm{g}$. The Bluetooth radio is a class 2 device with a radio range of around 10-20 meters. Each device records the results of the periodic device discovery and all data communications. In addition, the devices record details of the user's social profile and its evolution, and application level messaging. All traces are recorded constantly in text files on the device's SD memory card.All traces are time stamped based on the device clock and reported as a relative time in seconds since the start of the experiment. The device clocks are set manually to the same reference time at the beginning of the experiment.The types of object relationships were analyzed as shown in table 2 . Figure 2 shows the different types of device relationships. Table 3 shows the interest of 85 objects and their activities. Figure 3 gives the estimate of percentage of objects according to 7 interest groups.

Table 2: Types of object relationships

\begin{tabular}{||c||c||c||}
\hline \hline Type of relationship & Number of objects & Percentage \\
\hline \hline Parental Object Relationship & 65 & $76.47 \%$ \\
\hline \hline Co work Object Relationship & 35 & $41.17 \%$ \\
\hline \hline Co location Object Relationship & 50 & $58.82 \%$ \\
\hline \hline Ownership Object Relationship & 6 & $7 \%$ \\
\hline \hline Social Object Relationship & 85 & $100 \%$ \\
\hline
\end{tabular}

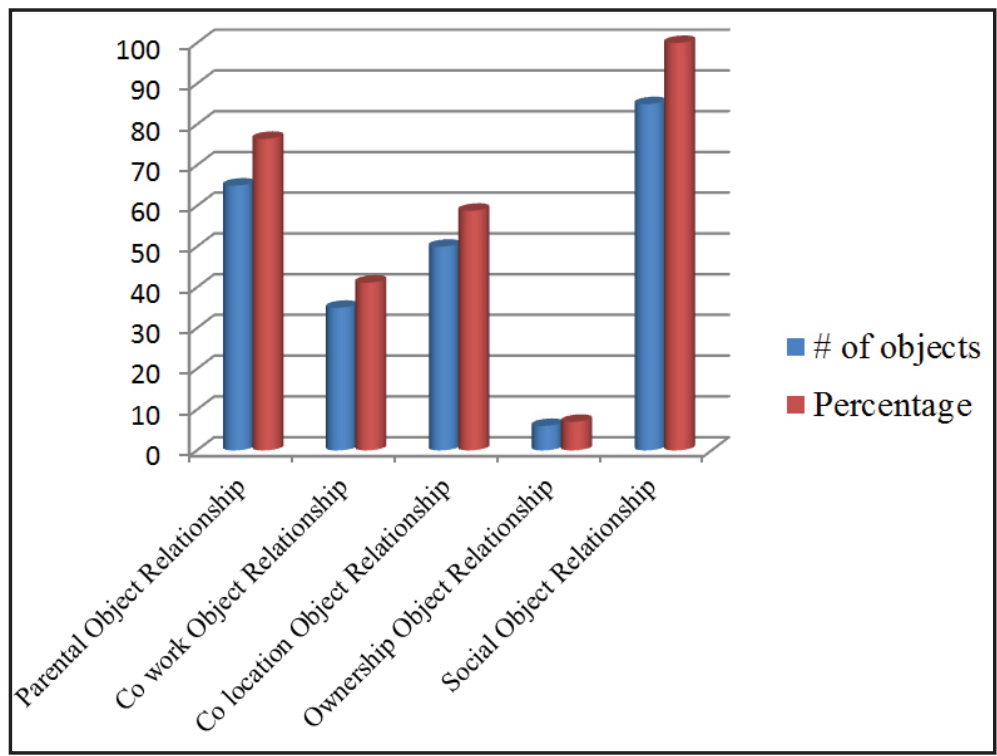


Table 3: Interests and activities identified among 85 objects

\begin{tabular}{|c||c||c||}
\hline Types of interest & \# of users & Percentage \\
\hline \hline Music & 7 & 8.23 \\
\hline \hline Sports & 4 & 4.7 \\
\hline \hline News & 42 & 49.41 \\
\hline \hline Facebook & 64 & 75.29 \\
\hline \hline Online shopping & 58 & 68.23 \\
\hline \hline Education & 34 & 40 \\
\hline \hline Others & 4 & 4.7 \\
\hline
\end{tabular}

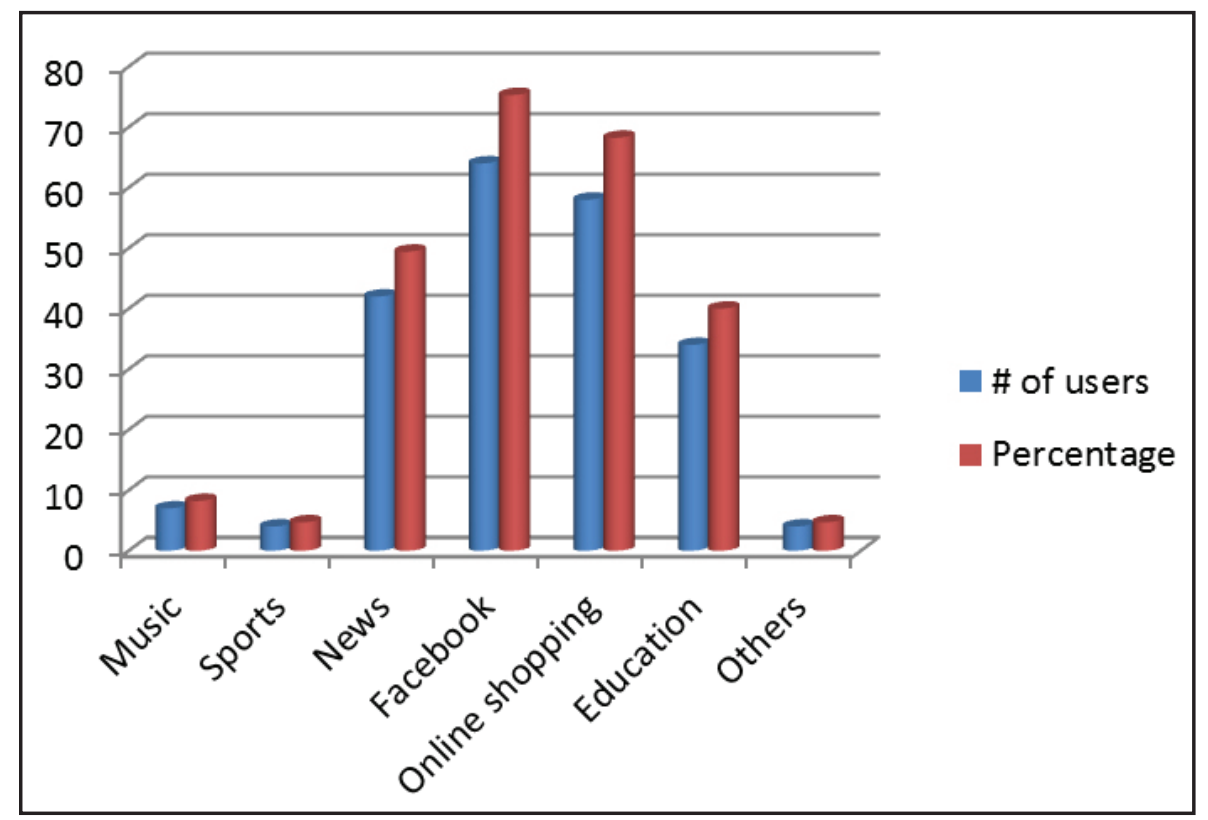

Figure 3: Objects classified according to interests

\section{CONCLUSION}

Social Internet of Things has integrated two new technologies namely Internet of Things and Social Networks. The SloT community is comprised of socially connected intelligent objects capable of collaborating with each other autonomously. This paper has introduced the notion of smart objects in loT becoming socially aware in the SloT world. The key enabling technologies of loT were discussed. The role, characteristics and relationship between social objects were identified and analyzed. A real time SloT environment was build comprising of 85 devices/objects. The relationship between objects and their activities were discovered. As a future work, we would like to focus on security and privacy issues in building a real time reliable SloT community.

\section{REFERENCES}

1) AtzoriLuigi, Antonio lera, and Giacomo Morabito 2011, SloT: Giving a social structure to the internet of things, Communications Letters, IEEE15.11: 1193-1195.

2) Atzori Luigi, Antonio lera, and Giacomo Morabito 2014, From smart objects to social objects: The next evolutionary step of the internet of things., Communications Magazine, IEEE 52.1, 97-105.

3) Kortuem, Gerd, et al 2010, Smart objects as building blocks for the internet of things, Internet Computing, IEEE 14.1 , 44-51.

4) Holmquist, Lars Erik, et al 2001, Smart-its friends: A technique for users to easily establish connections between smart artefacts, Ubicomp 2001: Ubiquitous Computing. Springer Berlin Heidelberg

5) Bleecker, Julian 2006, A manifesto for networked objects-cohabiting with pigeons, arphids and aibos in the internet of things. 
1) Mendes, Paulo2011, Social-driven internet of connected objects,Proc. of the Interconn. Smart Objects with the Internet Workshop.

2) Nazzi, Elena, and Tomas Sokoler 2011,Walky for embodied microblogging: sharing mundane activities through augmented everyday objects,Proceedings of the 13th International Conference on Human Computer Interaction with Mobile Devices and Services. ACM.

3) Kranz, Matthias, Luis Roalter, and Florian Michahelles 2010, Things that twitter: social networks and the internet of things, What can the Internet of Things do for the Citizen (CloT) Workshop at The Eighth International Conference on Pervasive Computing (Pervasive 2010)

4) Ning, Huansheng, and Ziou Wang 2011, Future internet of things architecture: like mankind neural system or social organization framework, Communications Letters, IEEE $15.4,461-463$.

5) Evangelos A, Kosmatos, Tselikas Nikolaos D, and Boucouvalas Anthony C 2011, Integrating RFIDs and smart objects into a UnifiedInternet of Things architecture,Advances in Internet of Things.

6) Jian, An, et al 2011, Nodes social relations cognition for mobility-aware in the internet of things, Internet of Things (iThings/CPSCom), 2011 International Conference on and 4th International Conference on Cyber, Physical and Social Computing. IEEE.
7) Atzori Luigi, et al 2012, The social internet of things (siot)-when social networks meet the internet of things: Concept, architecture and network characterization,Computer Networks 56.16, 3594-3608.

8) Kleinberg, Jon 2000 ,Thesmall-world phenomenon:Analgorithmicperspective,Proceedings of the thirty-second annual ACM symposium on Theory of computing. ACM.

9) MeenaKowshalya.A, M.L.Valarmathi 2015, Improved Network Navigability and Service Search in Social Internet of Things (SloT), International Journal of Research and Scientific Innovation, 2(11),77-75

10) Nitti Michele, Luigi Atzori, and Irena PletikosaCvijikj 2014, Network navigability in the social internet of things, Internet of Things (WFIOT), 2014 IEEE World Forum on. IEEE.

11) Farris Ivan et al 2015, Social Virtual Objects in the Edge Cloud,IEEE Cloud Computing 2.6, 20-28.

12) Fiske, Alan $P$ 1992, The four elementary forms of sociality: framework for a unified theory of social relations, Psychological review $99.4,689$.

13) A. Meena KOWSHALYA, M. L. VALARMATHI (2016), Community Detection in the Social Internet of Things Based on Movement, Preference and Social Similarity, Studies in Informatics and Control, 25(4), 499-506.

Paper sent to revision: 22.10.2016.

Paper ready for publication: 24.02.2017. 